\title{
Les Archives nationales du Québec : pour les affaires et la culture
}

\section{The Archives nationales du Québec: For Business and Culture Los archivos nacionales de Quebec: para los negocios y la cultura}

\section{Claude Minotto}

Volume 35, numéro 1, janvier-mars 1989

URI : https://id.erudit.org/iderudit/1028120ar

DOI : https://doi.org/10.7202/1028120ar

Aller au sommaire du numéro

Éditeur(s)

Association pour l'avancement des sciences et des techniques de la documentation (ASTED)

ISSN

0315-2340 (imprimé)

2291-8949 (numérique)

Découvrir la revue

Citer cet article

Minotto, C. (1989). Les Archives nationales du Québec : pour les affaires et la culture. Documentation et bibliothèques, 35(1), 3-11.

https://doi.org/10.7202/1028120ar

\section{Résumé de l'article}

Adoptée en décembre 1983, la Loi sur les archives du Québec assure la conservation, la mise en valeur et la diffusion des archives publiques, ainsi qu'un soutien aux services d'archives privées. Les Archives nationales du Québec sont chargées de l'application de la loi. L'auteur décrit le rôle important joué par cette institution et fait état des activités diverses mises en oeuvre par les neuf centres des Archives nationales du Québec.
Tous droits réservés $@$ Association pour l'avancement des sciences et des techniques de la documentation (ASTED), 1989
Ce document est protégé par la loi sur le droit d'auteur. L'utilisation des services d'Érudit (y compris la reproduction) est assujettie à sa politique d'utilisation que vous pouvez consulter en ligne.

https://apropos.erudit.org/fr/usagers/politique-dutilisation/ 


\title{
Les Archives nationales du Québec : pour les affaires et la culture
}

\author{
Claude Minotto* \\ Archives nationales du Québec \\ Montréal
}

Adoptée en décembre 1983, la Loi sur les archives du Québec assure la conservation, la mise en valeur et la diffusion des archives publiques, ainsi qu'un soutien aux services d'archives privées. Les Archives nationales du Québec sont chargées de l'application de la loi. L'auteur décrit le rôle important joué par cette institution et fait état des activités diverses mises en oeuvre par les neuf centres des Archives nationales du Québec.

\section{The Archives nationales du Québec: For Business and Culture}

Granted assent in December 1983, the "Archives Act" ensures the preservation, the display and the dissemination of public archives and advises private archives. The Archives nationales du Québec are responsible for the administration of the Act. The author describes the role of this institution and the varied activities of the nine regional centres.

\section{Los archivos nacionales de Quebec: para los negocios y la cultura.}

La ley sobre los archivos de Quebec adoptada en 1983 asegura la conservación, el valor y la difusión de los archivos públicos así como también un apoyo a los servicios de archivos privados. Los archivos nacionales de Quebec están encargados de la aplicación de la ley. El autor describe el papel importante de esta institución y menciona las diferentes actividades de los nueve centros de archivos nacionales de Quebec.
Qu'y a-t-il de commun entre les enregistrements des émissions de la série "Beau et Chaud", à Radio-Québec; les règlements municipaux de la ville où vous habitez; vos bulletins à la commission scolaire dont relève l'école que vous avez fréquentée, si vous êtes né une année finissant par 1 ou par 6 (ex. : 1941, 1946, 1951, 1956...); les rapports anatomopathologiques dans les centres hospitaliers; les états financiers d'HydroQuébec? II y a au moins ceci de commun à tout ce qui a été énuméré. Ce sont tous des documents faisant partie des $5 \%$ d'archives publiques qui seront conservées en permanence, d'abord pour leur utilité administrative et juridique, ensuite pour leur valeur historique. Les bulletins scolaires des élèves nés aux années 1 et 6 (ex.: 1941, 1946) sont conservés en permanence à titre d'échantillon représentatif de l'ensemble des bulletins dans une commission scolaire.

Par contre, environ $95 \%$ de l'ensemble des documents produits ou reçus par les organismes publics visés par la Loi sur les archives seront éliminés en vertu de cette loi, au terme d'une période de conservation au-delà de laquelle les documents en question n'ont plus d'utilité ni d'intérêt. D'ailleurs, qu'il s'agisse d'organismes publics ou privés, on estime à environ $5 \%$ seulement la proportion de leurs documents qui devrait être conservée en permanence. La proportion peut être plus élevée dans le cas d'organismes dont la vocation même est de créer des documents (ex.: Radio-Québec) plutôt que d'autres types de produits ou services (ex. : Société des Alcools du Québec, Alcan).

\section{Les archives, une ressource}

La Loi sur les archives du Québec fut adoptée par I'Assemblée nationale en décembre 1983. C'est une loi moderne et adaptée à notre milieu, issue d'une dizaine d'années de réflexion et d'expériences constamment mises à jour. Cette loi définit «document " et "archives " comme suit. Un document, c'est tout support d'information, y compris les données qu'il renferme, lisible par l'homme ou par machine, à l'exception des documents visés dans la Loi sur la bibliothèque 
nationale du Québec. Les archives sont l'ensemble des documents, quelle que soit leur date ou leur nature, produits ou reçus par une personne ou un organisme pour ses besoins ou l'exercice de ses activités, et conservés pour leur valeur d'information générale. Suivant cette définition qui tend à s'universaliser, les archives ne signifient pas seulement les documents anciens, mais désignent tous les documents y compris les plus récents. Mieux encore, un bon système d'archives, nous le verrons plus loin, tient compte des documents à venir.

Les documents d'archives se présentent donc sous formes très variées: textes manuscrits ou imprimés, cartes, plans, photographies, enregistrements sonores, films, rubans ou cassettes vidéo, microfilms ou microfiches, rubans ou disques "ordinolingues" (lisibles par ordinateur), etc. Ces documents sont des supports et des véhicules d'information. On les a créés pour consigner et communiquer des décisions, des ententes, des opinions, des découvertes, des témoignages, des images ou des interprétations de la réalité (photos, cartes, plans), etc. Les archives sont l'expression documentaire de la réflexion, de l'activité, de l'expérience. Dans tous les organismes et tous les milieux, pour le bénéfice des générations actuelles et de celles à venir, les archives favorisent le respect des droits, la continuité et la cohérence dans l'action, l'enrichissement du travail de création.

Ainsi, les archives d'un organisme, d'une entreprise ou d'une collectivité constituent une ressource nécessaire à l'efficacité de son administration, au succès de sa production, au développement de son histoire et de sa culture en tant que groupe. Le groupe accroîtra sa performance s'il réussit à mobiliser ses membres en leur transmettant ses valeurs culturelles, ses objectifs et l'élan historique qui en fait partie. Cette affirmation se confirme dans tous les domaines, que ce soit dans l'industrie, le commerce, les services ou dans la société en général.

Pour bien jouer ce rôle, les archives, comme toute ressource, doivent cependant être traitées et organisées de manière à être exploitées. Dans la société d'aujourd'hui, la multiplication des droits et obligations, le développement des communications et de l'économie elle-même, ainsi que plusieurs autres facteurs ont entraîné une production colossale et, dans plusieurs cas, une accumulation monstre de documents. Pour évoquer à la fois la puissance et le danger de ce phénomène, on parle de plus en plus souvent de "l'explosion documentaire» dans la société de l'information. Une enquête menée en 1984 auprès de 20 des 24 ministères et de 110 organismes gouvernementaux québécois visés par la Loi sur les archives a démontré les coûts excessifs engendrés par la conservation, dans des espaces à bureaux, d'une trop grande proportion de documents. Dans le secteur public, on aurait donc tendance à conserver trop et mal, puis à éliminer sans grand discernement ou à laisser périr les archives lorsque la situation devient incontrôlable. Le secteur privé éprouve des problèmes semblables.

La production des documents a requis un investissement dont la rentabilité peut être augmentée par un système approprié assurant en quelque sorte le "raffinage" de la masse documentaire.

\section{Un système $d$ 'archives}

Multiformes et innombrables, les documents sont créés parce que nécessaires. Toutefois, leur durée utile varie de l'un à l'autre. À l'image d'une certaine production contemporaine autodégradable, une très grande partie des documents produits est jetable.

Le système introduit par la Loi sur les archives du Québec s'appuie sur différentes notions archivistiques dont la "théorie des 3 âges". Suivant cette théorie, les documents traversent trois phases au cours de leur vie. Dans sa première phase, un document est "actif», c'està-dire qu'il est couramment utilisé à des fins administratives ou légales par l'organisme qui l'a produit ou reçu pour l'exercice de ses activités. Dans la deuxième phase de sa vie, on dit que le document est "semi-actif" parce qu'on y a recours à des fins administratives et légales seulement occasionnellement. En troisième et dernière phase, le document est qualifié «d'inactif» et, en général, il n'est plus utilisé à des fins administratives ou légales. "Actif, semiactif et inactif» sont des expressions qui ont peutêtre l'avantage de refléter l'éloignement progressif des documents vis-à-vis des strictes préoccupations administratives et légales des organismes. Du même coup, et c'est une bonne chose, ces expressions invitent les organismes au déplacement physique des documents hors des bureaux, vers d'autres lieux de conservation, sous la responsabilité de d'autres types de professionnels, lorsque changent l'âge et la vocation des documents. Par contre, "actif, semi-actif et inactif» sont des qualificatifs qui, malheureusement, peuvent avoir une connotation négative évoquant la passivité croissante et ne rendant pas justice à la valeur réelle des documents. Dans d'autres pays, on parle plutôt d'archives "courantes, intermédiaires, puis historiques".

Les documents n'ont évidemment pas tous la même durée utile, tant pour l'ensemble de leur 
vie que pour chacune de ses trois phases. Ainsi, selon le sujet et l'objet des différents documents, la période de conservation en phase active puis semi-active est extrêmement variable, allant de quelques jours à plusieurs années. Par exemple, les dossiers de règlements municipaux, qui sont considérés comme des documents essentiels, ont une période de conservation de 2 ans à l'état actif, après quoi ils passent directement en phase dite «inactive» où ils sont conservés en permanence. La période de conservation des dossiers de règlements municipaux en phase semi-active est donc nulle, tandis que les contrats municipaux pour services publics en sous-traitance (déneigement, par exemple) sont conservés en phase active pandant la durée du contrat; puis en phase semi-active, ils sont conservés pendant 28 ans à des fins légales, après quoi ils passent à l'état inactif où ils sont immédiatement détruits puisqu'on ne leur reconnaît spécifiquement aucun intérêt historique.

Dans le système des archives, on procède donc à l'évaluation de la durée utile des documents produits, et on programme à l'avance le cheminement de ces documents, soit vers l'élimination massive à court ou à moyen terme, soit vers la conservation permanente. Cette programmation se fait par l'établissement de l'instrument que l'on appelle le calendrier de conservation des documents. Dans son calendrier de conservation, un organisme précise les périodes d'utilisation et les supports de conservation de ses différents documents à l'état actif et semi-actif. Le calendrier indique également quels documents devenus inactifs sont conservés en permanence et lesquels sont éliminés. L'établissement et la mise en application du calendrier de conservation visent la rationalisation des processus et l'économie des interventions sur les documents, depuis leur création jusqu'à leur élimination, ou à leur exploitation permanente comme document historique.

Ce travail de programmation confère un caractère prospectif au système des archives. Dans une allocution prononcée lors du $X I^{e}$ Congrès international des Archives tenu à Paris en août 1988, le Président de la France, Monsieur François Mitterand, disait que les archives de demain ne seraient plus simplement ce qui reste quand le temps a passé. Les archives de demain seraient précisément ce que nous aurons prévu de mettre à la disposition de ceux qui nous suivront, d'une génération à l'autre. Établi sur des bases modernes, le système des archives, aujourd'hui, permet un service et des activités avantageuses.

\section{Un service d'archives}

La clientèle d'un service d'archives se divise en deux grandes catégories qui souvent se re- coupent; d'une part, les producteurs ou premiers propriétaires des documents, et d'autre part, les utilisateurs des documents, ceux que l'on appelle habituellement les "chercheurs". Selon les facteurs juridiques ou autres conditionnant l'accès aux documents, la clientèle des chercheurs sera plus ou moins large, et s'étendra au public général. Au Québec, le droit d'accès aux documents des organismes publics est accordé à toute «personne» tandis que dans d'autres pays, il est réservé aux citoyens du pays ou aux personnes «administrées" par le gouvernement qui fait la loi.

On dit que les clientèles des archives se recoupent car dans bien des cas, les représentants de l'organisme producteur des documents demeurent les plus grands utilisateurs de ces documents à des fins autres que strictement administratives ou légales.

Par un système qui enraie l'engorgement documentaire et facilite le repérage de l'information utile, le service d'archives offre à l'administration productrice de documents des économies et une efficacité accrues. II offre une ressource documentaire de choix pouvant contribuer en permanence à la performance et à la culture des clients qui ont accès aux documents.

Une société doit posséder et maîtriser les ressources documentaires qui lui permettent, rapidement et au moindre coût, de faire le bilan de son expérience dans tous les domaines et d'en tirer les meilleures leçons. Le service des archives doit veiller à ce que le même recours à l'information documentaire à jour demeure possible pour les années et les générations à venir. Le service des archives permet aux chercheurs de vérifier, de comprendre, de corriger, de planifier et de s'inspirer. Le service d'archives traditionnel avait déjà commencé à capitaliser le passé. Dans la mesure où il a réalisé une synthèse dynamique de l'action prospective autant que rétrospective, le service des archives d'aujourd'hui offre les sources de l'avenir. Comme d'autres produits, les documents traités et mis en valeur par les services d'archives apparaissent maintenant davantage comme les instruments et le reflet du progrès.

\section{Le rôle des Archives nationales du Québec}

Au moment de l'adoption de la Loi sur les archives du Québec par l'Assemblée nationale, en décembre 1983, le texte était précédé de très brèves notes explicatives qui n'ont pas été reproduites dans les éditions subséquentes de la loi. Le premier paragraphe de ces notes se lisait comme suit :

Ce projet de loi a pour objet d'assurer la conservation, la mise en valeur et la diffusion 
des archives publiques et d'apporter aux services d'archives privées une aide technique et financière.

\section{Dans le secteur public}

Pour un bon nombre d'organismes publics visés, le système introduit par la loi repose sur des notions archivistiques qui leur étaient auparavant peu connues ou inconnues. C'est pourquoi les auteurs de la loi ont mis beaucoup de soin à structurer le texte suivant la logique des opérations, à fournir les définitions nécessaires et à utiliser un langage accessible. C'est le ministre des Affaires culturelles qui est responsable de l'application de la loi mais la plupart des pouvoirs requis pour la mise en oeuvre des politiques et des règlements sont délégués aux Archives nationales du Québec (ANQ) par le biais du conservateur de cette institution.

La loi touche directement 3839 organismes publics au Québec. Leurs responsabilités vis-àvis de leurs archives varient selon le statut de l'organisme. Ainsi, les organismes du secteur public "centralisé" (les ministères et les organismes provinciaux qui en relèvent, ainsi que les tribunaux judiciaires) n'ont pas les mêmes responsabilités que les organismes du secteur public "décentralisé » (municipalités, établissements scolaires et de santé). La loi définit clairement les sept différentes catégories d'organismes publics concernés.

Tous les organismes publics ci-haut mentionnés ont une obligation commune. Ils doivent établir et tenir à jour un calendrier de conservation de leurs documents. Chacun des 3839 organismes publics doit également soumettre son calendrier de conservation pour approbation par les Archives nationales. Les organismes du secteur public "centralisé » doivent, une fois par année, verser aux Archives nationales les documents inactifs dont le calendrier de conservation prévoit la conservation permanente; tandis que les organismes du secteur public "décentralisé» doivent assumer eux-mêmes la gestion de leurs documents inactifs.

L'Assemblée nationale et les organismes dont elle nomme les membres doivent, pour leur part, transmettre leur calendrier aux ANO pour information seulement, et ils assument euxmêmes la gestion de leurs documents inactifs. Ce sont là d'importantes différences dans les responsabilités des organismes publics selon la catégorie à laquelle ils appartiennent en vertu de la Loi sur les archives.

D'autres différences portent sur l'étendue des responsabilités des ANQ vis-à-vis des organismes publics dans le cadre de politiques découlant de la Loi sur les archives. La loi prévoit que le ministre des Affaires culturelles adopte une politique de gestion des documents actifs et semi-actifs des ministères et de leurs organismes (secteur public "centralisé"). Pour les tribunaux et l'Assemblée nationale, le ministre propose une politique de gestion des actifs et semi-actifs, tandis que les organismes du secteur public "décentralisé» adoptent eux-mêmes leur propre politique, le ministre pouvant les conseiller à cet effet.

La politique de gestion des documents actifs des organismes publics visés au paragraphe 1 de l'annexe de la loi (ministères et organismes gouvernementaux dits "centralisés") a effectivement été adoptée par les Affaires culturelles en août 1985. Cette politique a fait progresser les organismes visés en reconnaissant à la gestion des documents un statut semblable à celui attribué à la gestion des autres ressources et en destinant le mandat de la gestion des documents à une unité administrative dont c'est la principale fonction, lorsque la taille de l'organisme le requiert. La politique adoptée précise en outre le rôle conseil des ANO en l'orientant sur l'aide à la préparation des calendriers de conservation, le choix de systèmes et de logiciels pour la gestion des documents, l'identification et la gestion des documents essentiels.

La politique de gestion des documents semiactifs adoptée par les Affaires culturelles à l'été 1988 préconise le regroupement des services de garde des documents semi-actifs des ministères et organismes sur une base régionale. Le Centre de Québec des Archives nationales gère, depuis 1984 déjà, un Centre de documents semi-actifs du Gouvernement.

En ce qui a trait à la gestion des documents inactifs, la loi confie aux Affaires culturelles la responsabilité plus étendue d'adopter une politique pour tous les organismes publics du Québec, quelle que soit leur catégorie. Le conservateur des ANO devra coordonner la mise en oeuvre de cette politique, en surveiller l'application et conseiller les organismes.

La Loi sur les archives du Québec possède quelques caractéristiques particulières qui en favorisent le succès. D'abord, la responsabilité d'approuver les calendriers de conservation n'est pas mitigée. Cette autorité sur les calendriers des 3839 organismes publics (excluant l'Assemblée nationale) est clairement située aux Archives nationales. Deuxièmement, la loi prévoit des échéances précises pour l'établissement des calendriers. Ces dispositions commandent l'engagement et la rigueur de la part des intervenants. Pour faciliter et accélérer le travail, les Archives nationales du Québec, en collaboration 
avec des comités distincts, chacun d'eux étant représentatif d'une catégorie d'organismes publics, ont mis au point et diffusé une quinzaine de recueils de délais de conservation de documents communs. Chaque catégorie d'organismes peut s'inspirer du recueil la concernant pour l'élaboration d'une partie de son calendrier. Ainsi, il existe un recueil pour les ministères, un pour les municipalités, un pour les hôpitaux, etc.

Offrant de multiples avantages administratifs autant que culturels, la Loi sur les archives a été fort bien accueillie. L'introduction, enfin, d'un système d'archives a été vivement appréciée, particulièrement par les organismes où il n'y en avait aucun. Les statistiques témoignent des progrès rapides de la mise en application de la loi. Au 31 mars 1988, sur 3839 calendriers de conservation prévus, 2402 , soit $63 \%$, ont déjà été soumis pour approbation, et 1212 ont été analysés et approuvés. Les ANQ prévoient que d'ici avril 1990, tous les organismes publics auront fait approuver leur calendrier.

\section{Dans le secteur privé}

Partout au Québec, d'importantes archives sont de nature privée et demeurent propriété privée: archives d'entreprises, archives de sociétés à but non lucratif, archives d'individus et de familles, archives d'institutions qui laissent leur marque sur le Québec ou sur une de ses régions. La loi charge les Archives nationales du Québec de promouvoir la conservation et l'accessibilité des archives privées.

Depuis de nombreuses années, les ANO acquièrent (principalement par voie de don) des fonds d'archives privées d'intérêt régional et national. Cette prise en charge directe se poursuivra, d'autant qu'elle repose maintenant sur la concertation entre les ANO et d'autres services d'archives concernés. Mais les ANO veulent aussi, par différents moyens, favoriser le développement d'organismes privés compétents qui assureraient la conservation et l'exploitation des archives privées. Par son programme d'aide financière, les ANQ apportent leur soutien à des projets de traitement et de diffusion d'archives privées. La Loi sur les archives propose un nouveau moyen pour appuyer le développement de services d'archives privées et pour reconnaître en même temps la qualité du travail dans ces services.

En effet, une personne ou un organisme peut demander au ministre des Affaires culturelles "d'agréer» son service d'archives privées. L'«agrément " accordé par le ministère à certains services d'archives comprendrait d'importants avantages tels que l'accès prioritaire à l'aide financière et aux services-conseils professionnels et techniques des ANO.

\section{Le rôle incitatif et le rayonnement des ANO}

L'adoption et la mise en application de la Loi sur les archives ont eu des effets d'entraînement. Des programmes universitaires d'enseignement de l'archivistique (Université de Montréal, Université du Québec à Montréal, Université Laval) se sont développés en partie grâce à la collaboration initiale d'archivistes des ANQ qui ont accepté des charges de cours. Ces programmes ont d'abord enregistré une importante clientèle étudiante issue principalement d'organismes publics directement concernés par la loi. Quelques grandes sociétés d'État québécoises et canadiennes comptent des étudiants ou diplômés en archivistique parmi les membres de leur personnel, par exemple, Hydro-Québec, RadioCanada, Téléglobe Canada, la Société du Port de Montréal. Les étudiants font des stages pratiques dans les milieux de travail et des diplômés accèdent à de nouveaux emplois créés. On les retrouve dans une variété d'organismes publics et privés tels que la Bourse de Montréal, la compagnie Oerlikon, Canadair, CP Rail, Alcan, la Société de transport de la Communauté urbaine de Montréal, l'École Polytechnique, les municipalités de Saint-Hubert, de Pierrefonds et de Lachine, etc.

La mobilisation suscitée au Québec par la Loi sur les archives a attiré l'attention de responsables d'archives d'autres régions du Canada et du monde.

La Loi du Québec a influencé l'évolution du projet de loi fédéral canadien sur les archives, loi qui fut adoptée par Ottawa en juin 1987. L'État de New York a adopté, en août 1987, une loi sur les archives des gouvernements locaux et récemment, l'archiviste de l'État de New York communiquait avec le conservateur des ANQ afin d'organiser une rencontre à laquelle participeraient différents intervenants newyorkais au sujet de l'expérience du Québec dans l'application de la législation sur les archives. Deux représentants de haut niveau des Archives d'URSS ont visité les Archives nationales du Québec en novembre 1987. La Tunisie a adopté une loi sur les archives en s'inspirant de l'approche québécoise. Le Conseil international des Archives, organisme réunissant plus de 130 pays, tiendra son congrès quadriennal de 1992 à Montréal. Ce XII Congrès international des Archives fera suite à celui de Paris (1988), de Bonn (1984), de Londres (1980), de Washington (1976), de Moscou (1972), etc. Le Congrès international de 1992 attire déjà d'autres groupes importants dont la Society of American Archivists (SAA) qui a décidé de tenir 
son propre congrès annuel de 1992 à Montréal à la suite du congrès international. L'Association des archivistes du Québec (AAQ) et l'Association of Canadian Archivists (ACA) seront également présentes. Montréal attend donc en 1992 plus de 2000 spécialistes des archives venus de tous les coins du monde.

Lors du XIe Congrès international des Archives qui s'est tenu à Paris en août 1988, les directeurs nationaux des Archives des pays francophones se sont réunis pour discuter de leurs intérêts communs d'un projet d'association, comme il en existe pour d'autres institutions et professions à travers la francophonie. Un groupe de réflexion a été constitué, et son mandat consiste à préciser les objectifs de l'association à venir, à préparer les statuts et, déjà, à planifier un programme d'action qui sera soumis aux membres.

Le groupe de réflexion comprend les cinq personnes suivantes: Jean-Wilfrid Bertrand (directeur des Archives nationales d'Haïti), Moncef Fakh Fakh (directeur des Archives nationales de Tunisie), Robert Garon (conservateur des Archives nationales du Québec), Saliou M'Baye (directeur des Archives nationales du Sénégal) et Paule René-Bazin (responsable des relations internationales, Archives nationales de France). La direction du groupe a été confiée à Robert Garon.

\section{Le réseau et les ressources des ANQ}

Depuis la création du Bureau des archives du Québec en 1920, jusqu'en 1970, le service des archives était centralisé à Québec même. À compter de 1971, les ANQ ont graduellement mis sur pied leur propre réseau de centres d'archives, pour couvrir le vaste territoire du Québec et mieux servir les organismes et les chercheurs de chaque région. Les ANO comprennent aujourd'hui neuf centres d'archives établis dans les villes suivantes: Sainte-Foy, pour la région de Québec; Montréal, depuis 1971; Trois-Rivières (1975); Hull et Chicoutimi (1978); Rimouski et Sherbrooke (1979); Rouyn-Noranda (1980); Sept-Îles (1981).

Pour l'ensemble des neuf centres, les ANO disposent d'un personnel de 124 employés permanents et d'environ 15 postes pour contractuels. En 1987-1988, le budget total des ANO s'établissait à 5135000 s dont 4167000 \$ étaient affectés à la rémunération.

\section{Objectifs et priorités des ANQ}

Pour la tenue du XII Congrès international des Archives à Montréal en 1992, on a mis sur pied un comité organisateur dont le caractère reflète l'importance de cet événement majeur. Les Archives nationales du Québec furent à l'origine même des premières initiatives des Archives nationales du Canada en vue d'obtenir pour Montréal, en 1992, ce congrès quadriennal du Conseil international des Archives (CIA). Les ANO sont donc un partenaire important dans l'organisation du congrès. Le conservateur des ANO assume la fonction de vice-président du comité organisateur tandis que l'archiviste national du Canada en assure la présidence.

Ainsi engagées vis-à-vis de ce grand rendezvous de la profession, les ANO misent sur une excellente préparation. Les objectifs de travail, les priorités et le plan d'action des ANQ ont fait l'objet d'une planification rigoureuse, avec la participation de tous les employés. II en résulte un programme d'ensemble intitulé Vers 1992. Ce programme comprend 25 objectifs institutionnels rattachés à l'un ou l'autre des neuf thèmes suivants :

Thème 1: La gestion des documents administratifs des ministères et organismes.

Thème II: La gestion des documents inactifs confiés aux ANQ.

Thème III: Les calendriers de conservation.

Thème IV: La gestion des archives historiques des organismes publics décentralisés.

Thème $V$ : La gestion des archives privées.

Thème $\mathrm{VI}$ : La formation et le perfectionnement.

Thème VII: La modernisation de l'institution.

Thème VIII: Les services à la clientèle utilisatrice.

Thème IX: Les expositions - Les publications.

Les 25 objectifs articulés autour de ces neuf thèmes mais fixés par ordre de priorité seront poursuivis à travers une centaine d'actions bien précisément identifiées et dont la réalisation s'échelonne sur la période 1988-1992.

Parmi les objectifs prioritaires, nous retrouvons, en tête de liste, l'approbation de tous les calendriers de conservation des documents des organismes publics centralisés et décentralisés. Tel que mentionné plus haut dans le texte, les ANO prévoient atteindre cet objectif pour la fin de 1990. II est également prévu que des méca- 
nismes de suivi, de contrôle et d'évaluation soient mis au point, toujours en collaboration avec les organismes visés, pour assurer l'efficacité des calendriers.

L'application des principales politiques découlant de la loi occupe un rang élevé dans l'ordre des priorités des ANO : politique de gestion des documents actifs, semi-actifs et inactifs des organismes gouvernementaux, politique en matière d'archives privées.

Aux niveaux national et international, les services d'archives souhaitent une plus grande standardisation des pratiques à l'intérieur des principales fonctions archivistiques : acquisitions d'archives, tri, sélection, description, traitement, diffusion, conservation. Les ANO ont établi et adopté onze normes pour les acquisitions, le tri et la sélection d'archives de différents types et supports. Ces normes ont été imprimées et diffusées. Dans le programme Vers 1992, une série d'actions a été planifiée pour compléter ce travail. Pour 1992, l'objectif visé consiste à avoir produit, expérimenté, appliqué et diffusé les normes et procédures archivistiques des ANO.

Le sujet de chaque norme à produire a déjà été identifié. L'ensemble des normes et procédures fera l'objet d'une publication majeure.

Le développement de l'informatique et de la bureautique figure également parmi les priorités et les dossiers majeurs. Un schéma directeur a été réalisé et présenté. Les ANQ continuent à doter leurs centres des équipements informatiques facilitant l'application quotidienne du plan de classification et du calendrier de conservation des documents de l'institution. Le schéma directeur comprend aussi la révision du système SAPHIR qui serait transformé, d'ici trois ans, en système interactif à accès direct pour le personnel et pour les chercheurs à travers tout le réseau des ANO.

\section{Les archives judiciaires}

Autre vaste chantier des ANQ, le domaine des archives judiciaires. Pendant longtemps, surtout dans le district judiciaire de Montréal, le sort des archives judiciaires produites depuis 1763 a vivement inquiété, avec raison, le milieu des archives et de la recherche historique. Périodiquement, les chercheurs universitaires ont dénoncé les conditions de conservation lamentables et parfois périlleuses des archives judiciaires dans plusieurs Palais de Justice, ainsi que les énormes difficultés d'accès à cette documentation. De son côté, l'administration judiciaire a été sérieusement incommodée et taxée par le maintien de ces masses documentaires en très forte croissance, la pratique traditionnelle des tribunaux ayant été de conserver toutes les pièces judiciaires de tous les dossiers. De nos jours, plus de 400000 dossiers de toutes natures sont ouverts annuellement dans les greffes de nos cours. A eux seuls, les documents judiciaires anciens, ceux qui ont déjà plus de 30 ans d'âge, sont sommairement évalués à quelque 25 kilomètres linéaires, soit plus de 750 tonnes de documents! L'expression «le poids de l'histoire» trouve ici une signification lourde de conséquence.

La Loi sur les archives ayant reconnu la très grande importance de ce secteur et l'urgence d'y intervenir, les tribunaux comptent parmi les organismes publics devant établir et tenir à jour un calendrier de conservation de leurs documents. Plusieurs facteurs, toutefois, font de cette opération un défi de taille: d'abord, la question des droits des justiciables qui sont consignés dans certains types de documents judiciaires; puis, les traditions séculaires qui ont créé la situation complexe que nous connaissons aujourd'hui, mais qui, malgré tout, ont sauvegardé de précieuses séries de documents qu'il faut pouvoir cerner à l'intérieur de la masse; ensuite, le problème de la méconnaissance des besoins réels des chercheurs et des tribunaux (les producteurs de ces documents); enfin, les moyens de l'État pour organiser la sélection, la description, la conservation et la diffusion des archives judiciaires qui auront été «jugées» d'intérêt historique.

Confrontées à ces problèmes, les Archives nationales, avec l'accord et l'appui de la Direction générale des services judiciaires du ministère de la Justice du Québec, ont proposé, dans un mémoire transmis aux sous-ministres de la Justice et des Affaires culturelles, de créer un comité interministériel représentatif des milieux concernés, lequel serait chargé, après analyse des problèmes, de soumettre ses recommandations aux sous-ministres mandataires. La proposition des Archives nationales a été accueillie très favorablement et un comité a été mis sur pied.

Ce comité de haut niveau regroupe neuf personnes représentant des producteurs, des gestionnaires et des conservateurs ainsi que des utilisateurs d'archives judiciaires. Les Archives nationales du Québec et la Direction générale des services judiciaires du ministère de la Justice travaillent donc à ce comité en collaboration avec des représentants de tous les milieux concernés, soit la Magistrature, le Barreau, la Chambre des notaires et les milieux de la recherche du Québec.

Selon les évaluations soumises au comité, l'ensemble des documents judiciaires produits de 1764 à 1985 et conservés à ce jour totalise 90 kilomètres linéaires, dont plus du tiers semble être constitué de documents inactifs. 
Une des premières tâches du comité fut d'établir des principes et des critères qui ont permis d'élaborer les calendriers de conservation des documents judiciaires. Le comité s'est interrogé sur la véritable portée de la notion de "cours d'archives" présente dans la Loi sur les tribunaux judiciaires qui a conduit, jusqu'à ce jour, à la conservation intégrale des documents. Une étude du régime juridique applicable à la conservation des dossiers judiciaires a été réalisée pour le comité. Cette étude a démontré que le processus de conservation, même sélectif, prévu dans la Loi sur les archives ne porte nullement atteinte à la notion d'indépendance des tribunaux dans la mesure où les juges en chef demeurent responsables d'établir le calendrier de conservation déterminant à quel moment la conservation des archives n'est plus nécessaire pour les fins de I'accomplissement des fonctions judiciaires. L'hypothèque imposée jusqu'à maintenant par le concept de "cours d'archives" semble donc clairement levée.

Le comité a également commencé à définir des critères qui faciliteraient, d'une part, la sélection, pour conservation permanente, des séries documentaires d'intérêt historique et, d'autre part, l'élimination, sauf spécimens, des masses de documents sans intérêt historique appréciable. Ainsi, le comité croit nécessaire de conserver intégralement les registres de jugements et les instruments de repérage tels que les plumitifs et les index. Les séries documentaires dont le contenu s'avère unique pour la recherche seraient aussi conservées intégralement jusqu'à une date charnière qui sera vraisemblablement établie autour de 1920. C'est à compter de cette date que commencent l'inflation documentaire et la diversification des sources d'information. On peut alors envisager l'échantillonnage statistique auquel pourrait s'ajouter une sélection qualitative dont les critères restent à établir.

Déjà, des chercheurs explorent les séries d'archives judiciaires versées récemment aux ANQ et ils suivent attentivement l'évolution des affaires dans ce secteur. Le programme du congrès annuel de I'Institut d'histoire de l'Amérique française en octobre 1987 incluait un exposé sur la nature et l'accessibilité des archives judiciaires conservées aux ANOM. Quelques mois plus tard, une trentaine de chercheurs spécialisés ont participé à une table ronde organisée par I'Université du Québec à Montréal sur la sélection des archives judiciaires pour conservation permanente.

Enfin, toujours parmi les dossiers majeurs des $A N Q$, le projet pour l'aménagement définitif du Centre d'archives de Montréal (ANOM) retient l'attention des autorités ministérielles. On se souvient que durant I'hiver 1987, les ANOM avaient dû quitter d'urgence leurs locaux du 100 , rue Notre-Dame Est (Édifice Ernest-Cormier), suite à des problèmes survenus dans cet édifice en cours de rénovation. Les ANOM se sont alors installées provisoirement au 1945 rue Mullins, mais cette installation provisoire ne leur permettra pas de remplir leur mandat durant de nombreuses années. C'est pourquoi le ministre des Affaires culturelles a exprimé la volonté du ministère de relocaliser, d'ici 1992, les ANOM au centre-ville, en les installant de manière à ce qu'elles puissent y assurer à long terme le service des archives auprès des clientèles nombreuses et diversifiées de la région de Montréal.

\section{Les opérations courantes et l'apport culturel original}

Les ANO acquièrent à chaque année maintenant environ un kilomètre linéaire de documents pour conservation permanente. En 1988, la quantité totale d'archives historiques conservées aux ANQ s'élève à environ 27 kilomètres linéaires de documents écrits, auxquels s'ajoutent environ 620000 cartes et plans, cinq millions et demi de photographies et images fixes, 28700 enregistrements sonores et près de 11000 documents sur film ou ruban vidéo.

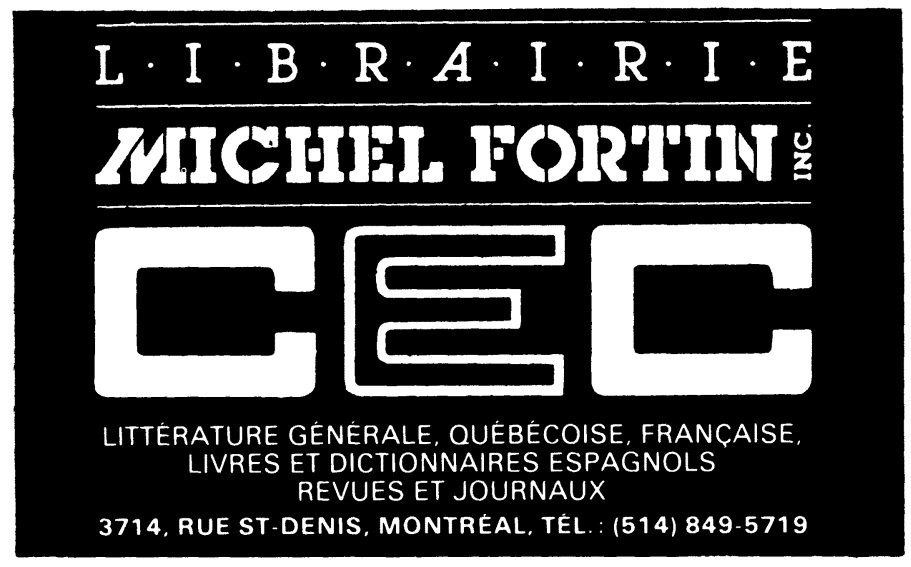


Dans les neuf centres des ANQ, on enregistre au total plus de 35000 visites de chercheurs et plus de 100000 demandes de consultation de documents. Les centres d'archives accueillent des visiteurs en groupes, près de 150 groupes par année. Enfin, les ANQ présentent près de 30 expositions de documents par année et quelquesunes de ces expositions voyagent d'un centre à l'autre.

Le Centre d'archives de Montréal est doté d'un atelier de restauration de documents qui effectue des travaux pour tous les centres du réseau des ANO.

C'est dans les activités spécifiques récentes de chacun des centres des ANO que l'on reconnaîtra et que l'on appréciera l'originalité de l'apport culturel des archives: acquisition des archives de J.G. Lacombe, photographe professionnel à Rimouski (1940-1970); répertoire du fonds d'archives de l'Association canadienne de la jeunesse catholique (1904-1960) et index des nouvelles télévisées par CKRS-TV, (1963-1985), au Centre d'archives de Chicoutimi; étroite collaboration du Centre d'archives de Québec à l'exposition consacrée au photographe Livernois, dans le cadre des événements qui ont souligné les 150 ans de la photographie à Québec; indexation des contrats de mariage dans les archives notariales de la région de Trois-Rivières, pour la période 1647-1885; exposition «Visages d'Alfred Desrochers" (1905-1978) présentée à Sherbrooke par le Centre d'archives de l'Estrie; premier versement d'archives de Radio-Québec au Centre d'archives de Montréal, et collaboration de ce centre à l'exposition "Revue-mode de la crise à l'après-guerre"; exposition sur le "Patrimoine architectural du Pontiac» (1820-1978) présentée par le Centre d'archives de l'Outaouais : "Visages d'autrefois", une exposition retraçant l'évolution des quartiers de Rouyn-Noranda des années 1920 aux années 1950; présentation de l'exposition «Industries sur la Côte-Nord», au centre de Sept-Îles, illustrant les activités de cinq compagnies dans les secteurs de l'électricité, de l'aluminium, du fer, des pâtes et papiers et de la pêche.

Ces exemples sont tirés des nombreuses activités annuelles des neuf centres d'archives des ANQ, une institution qui se prépare activement au XII Congrès international des Archives à Montréal en 1992.

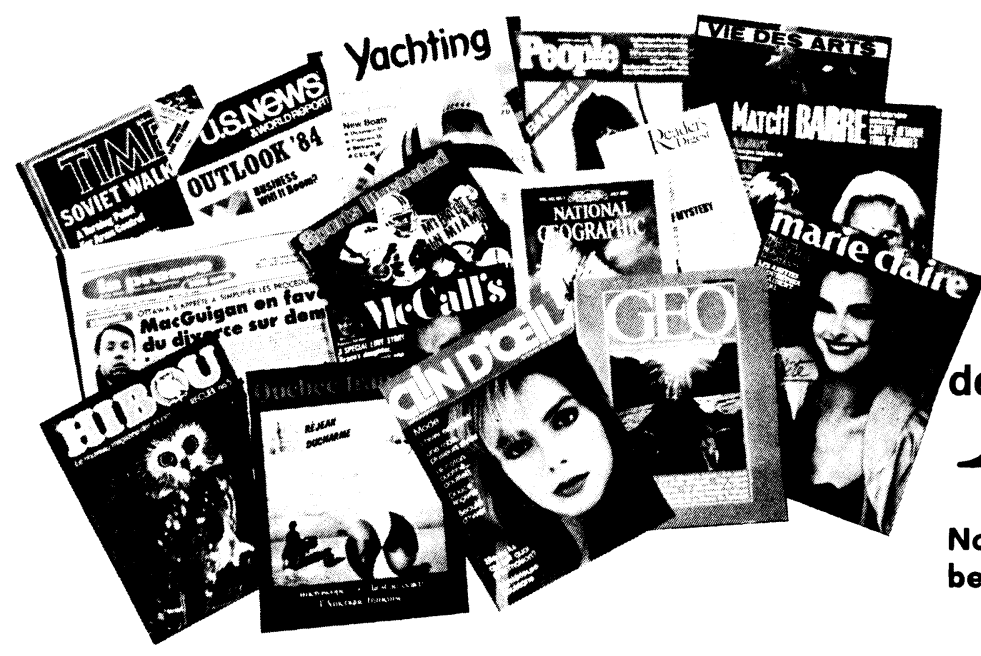

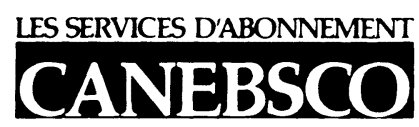

SIX BOUL. DESAULNIERS

SUITE 308

ST. LAMBERT, QUE J4P IL3

(514) 672-5878

Ligne directe pour Québec:

(800) 361-7322
SERVICE AU QUÉBEC:

- Centre de traitement

à St.-Lambert

-Equipe spécialisée

- Banque de données

- Avis professionnels

-Représentant des ventes 